\title{
Analysis of Conflict Diffusion Over Continuous Space
}

\author{
Claire Kelling and YiJyun Lin
}

\begin{abstract}
This study illustrates an innovative application of methods in spatial statistics to study the diffusion of conflict events. We investigate how spatial processes of conflict events vary with different characteristics of the events and actors involved in the events. Actor-level attributes have often been ignored in existing empirical studies, which could lead to insufficient modeling of conflict processes and patterns. Due to recent technological and systems advances, conflict events can now be analyzed using data measured at the event (point) level, rather than relying on aggregated units. Our research contributions are twofold. First, through the case of South Sudan, we demonstrate how intensity and covariance functions, defined by the log-Gaussian Cox process model, can be used to explore the complex underlying diffusion mechanism under various characteristics of conflict events. Second, our findings add to the explanation for the process of conflict diffusion. Our analysis reveals that battles with territorial gains for one side tend to diffuse over larger distances than battles with no territorial change, and that conflicts with longer duration exhibit stronger spatial dependence.
\end{abstract}

Keywords Point process - Continuous space models · Diffusion · Conflict duration $\cdot$ Spatial statistics

\section{Introduction}

Prolonged civil conflict and war as well as humanitarian crises occur frequently and are widespread in the African continent. This study selects the case of South Sudan as an example to illustrate how our methodological approach can improve understanding of conflict diffusion. Certainly, research findings drawn from a single

\footnotetext{
C. Kelling $(\bowtie)$

Department of Statistics, Pennsylvania State University, State College, PA, USA

e-mail: ckelling@vt.edu

Y. Lin

Department of Political Science, University of Nevada, Reno, Reno, NV, USA

(C) The Author(s) 2020

E. Deutschmann et al. (eds.), Computational Conflict Research,

Computational Social Sciences, https://doi.org/10.1007/978-3-030-29333-8_10
} 
case study are difficult to generalize. Conflict processes in South Sudan, however, fit the methodological goal of this study because varieties of actors have engaged in different types of conflict. This could provide a rich dynamic for how conflict events spread around areas within the country given the capacity of actors. Moreover, lessons drawn from South Sudan could also shed light on the theory of conflict: this country's history of dependence indicates that diffusion of conflicts within a country can unfold even with peaceful agreement among contentious parties.

From South Sudan's independence in July 2011 up to December 2013, President Salva Kiir and opposition leader and former Vice President Riek Machar have successfully integrated rebel groups in the national army and gave money to hundreds of generals and soldiers. Some of these generals and soldiers have become ministers at the national or local level, occupying key roles in the 35 states in which South Sudan is now divided. This problem is further complicated by the fact that there are around sixty indigenous ethnic groups in South Sudan, and that its national income, typically oil, has been used to buy the loyalty of these generals and soldiers. The consequence is that South Sudan has been trapped into prolonged civil conflicts and wars since 2013 (GlobalConflictTracker, 2019), and has also become one of the most conflict-prone countries in the world, which has gained much attention by the international community (CrisisGroup, 2017). The civil conflicts and wars in South Sudan have caused approximately four million people to be internally displaced within South Sudan or to flee to neighboring countries. This phenomenon further exacerbates regional conflicts and humanitarian crises. Humanitarian access has been obstructed by the increasing intensity of active hostilities or inter-communal violence (OCHA, 2018).

The historical conflict process in South Sudan is, therefore, counter-intuitive for the following two reasons. First, it is expected to be extremely difficult to peacefully moderate conflicts in South Sudan due to the presence of multiple factions against the government and the ubiquitous nature of interconnected civil conflicts. Yet South Sudan was able to reach and sign a Comprehensive Peace Agreement (CPA) with Sudan in 2005 in attempt to end the 50 years of violent conflict. Second, the international community believes that working on peace deals between the government and opposition is the main solution to the prolonged conflict. However, such a peaceful agreement quickly failed to resolve local grievances and maintain peace, and the South Sudanese people increasingly believe that violence is a necessary mean to achieve peace (De Vries and Schomerus, 2017). Thus far, the international community is still puzzling over what policy tools can effectively deal with these massive conflict-driven crises. South Sudan's experience calls our attention because false solutions can be made if the international community fails to realize the fact that peace and conflict are two sides of the same coin.

A conventional approach to building conflict theory and testing associated hypotheses has primarily concentrated on national-level institutional characteristics and state motivations or capability of going to war by using country-year aggregate data (Hegre and Nygård, 2015; Hegre, 2014; Maves and Braithwaite, 2013; Hoeffler, 2012; Braithwaite, 2010). This approach has provided insights for understanding the persistent nature of conflict in many countries. However, this approach cannot 
capture whether and how varying types of conflict and actors involved are spatially linked to each other in ways that exacerbate conflicts within a country over time. On the one hand, country-level institutional characteristics or aggregate economic conditions are often time-invariant or slow-moving factors. As a result, it is hard to justify the condition under which time-invariant variables might influence dynamics of conflict events. On the other hand, although domestic conflict events and the formation of rebel groups were believed to be inevitable in countries where governments are corrupt or political institutions are weak (Collier et al., 2009; Fearon and Laitin, 2003), non-state actors may have different grievances, demands, and means of holding conflict. Therefore, countries could experience distinct and various internal conflict processes and outcomes.

Moreover, conflict scholars have not reached consensus with regard to whether violence against civilians emerges as irrational random attacks, as a consequence of the tension between ethnic groups, or as an instrument for powerful groups to reach their political and military goals (Valentino, 2014, see also Salvi et al. in the chapter "Violence Against Civilians" in this book). Although it is likely that a country's political characteristics can drive civil unrest, many conflict events in Sudan were driven by actors' local demands and grievances. Some of these conflicts diffuse or spread from their initial locations, while other conflicts do not spread out across regions within a country's territory (Raleigh et al., 2010). Thus, country-level factors fail to explain spatial patterns of conflict diffusion within a country, especially due to actor types such as whether they are state, non-state, or civilian actors.

Fortunately, the recent advances in technological systems and methodologies enable conflict scholars to tackle actor-level characteristics as a mechanism by using conflict data at the event (point) level, rather than relying on aggregated units, which can better capture local dynamics and spatial variations in conflict. In this paper, we make use of disaggregated spatial data, together with continuous space models, through which we shift from the conventional monodic, state-based approach to an actor-centered approach to reexamine different types of conflict and test conflict diffusion on the local level. This study also deviates from the traditional approach of analyzing the effect of actor-level characteristics on conflict events at the country level, such as actors' ethnicity, and their capabilities of appealing to violence (Cederman and Gleditsch, 2009; Buhaug and Gleditsch, 2008; Harbom et al., 2008; Toft, 2005), by incorporating the spatial and temporal process of conflict as well as actor-level characteristics at the conflict point level. This analytical strategy fills a gap in the literature, in that it helps to answer what causes some differences in conflict diffusion patterns. In some cases, small conflicts scale up to a violent conflict event, while in other cases they do not and these conflict events may or may not further spread around areas within a country (Hoeffler, 2012; Buhaug et al., 2011). So far, only few empirical works analyze conflict in continuous space (Zammit-Mangion et al., 2012). Our study builds on the literature of conflict point processes over continuous space through detailed analysis of duration length, actor types, conflict types, and temporal elements.

In this study, we analyze five types of conflict events: "Battle-Government regains territory," "Battle-No change of territory," "Battle-Non-state actor over- 
takes territory," "Riots/protests," and "Violence against civilians." These types of conflict are closely related to the political instability and contentious state accompanied by the history of South Sudan being an independent country since 2011. In some cases, civil conflicts may be smaller episodes of a larger civil war, while others are not. By analyzing these event types in detail, this study captures scenarios where actors have a relatively equal military power (e.g., the first three categories of conflict) versus actors have an unequal capacity to appeal to violence (e.g., the last two categories), and differences in the spatial diffusion mechanism of conflict events by these event types.

By incorporating different types of conflict events and actors involved in conflict, and modeling the conflict process of these events, this study is able to capture differences in patterns of conflict diffusion in South Sudan. This analytical strategy enables us to assess a range of conflict dynamics because large-scale violence encompasses episodes of random killing, violence against civilians as well as the use of selective or indiscriminate violence (Koc-Menard, 2006). These different types of conflict reveal various purposes and/or goals pursued by the actors engaged in conflicts, such as advancing military interests, social identity, or political loyalty (Schwartz and Straus, 2018; Valentino, 2014; Balcells, 2011). We believe it is theoretically meaningful to reexamine different types of conflict based on the actor-level characteristics because actors involved in these events vary with their motivation, strategic behaviors, and capabilities of appealing to violence, which could further lead to distinct conflict processes and patterns. Distinguishing between state, non-state, and civilians as actors is theoretically meaningful because these actors directly determine where and when conflicts might take place as well as the condition under which their motivations, capabilities, and strategic responses might (or might not) lead to full-blown civil war (Cunningham et al., 2013).

Lastly, we study the difference in diffusion mechanisms by the duration of the conflict event. In many ecological and epidemiological models as well as models on crime, the data that is collected is simply an event or observation at a certain point and time (Wang et al., 2016; Liang et al., 2008; Best et al., 2000; Sparks, 2011). However, this may not be true of conflict data, where an event can have a wide range in its duration. Some events merely last for 1 day, while other events persist for several days or weeks at certain time periods. Therefore, we differentiate between conflict events that last only 1 day with conflict events that last longer than 1 day in our study.

The paper is organized as follows. In the next section, we summarize relevant research in three categories: empirical studies on the spread of conflict and wars, methodological studies on models for grid data, and continuous space models. Section 3 summarizes the data we use in this study, and in Sect. 4 we describe the methods and present the results, comparing conflict events based on time, conflict type, and actors involved. We conclude with a summary and some caveats in Sect. 5. 


\section{Related Work}

\subsection{Empirical Studies on the Diffusion of Conflict}

When it comes to spatial modeling, scholars have often either focused on spatial dependence alone or spatial heterogeneity by using distances between conflict events or borders between cities and states (Anselin and O'Loughlin, 1990). Typically, empirical works on modeling space or location for conflict events have relied on modeling the covariance structure directly, or non-constant error variances in a regression model (Anselin and Baltagi, 2001; Starr, 2003; Ward and Gleditsch, 2002). Thus far, there is no consensus yet with regard to what methods can better identify empirical patterns of conflict diffusion in a robust way, and there are no concrete theoretical linkages pointing out how spatial effects might influence the diffusion of conflict. For example, there could be demonstration effects or diffusion effects. The former refer to the effect derived from either organized or spontaneous collective action. The latter often rely on a larger scale and latent learning process, through which rebel groups learn by observing others. There could also be contagion effects, as rebels move over borders to incite rebellion by their co-ethnics in neighboring countries. Even though methods for robustness tests are available (Bera et al., 2019; Penghui et al., 2015), they are ad hoc. Therefore, it is unclear whether differences in empirical results are due to estimation methods, measurement, data sources, and/or spatio-temporal coverage (Hegre and Sambanis, 2006).

Schutte and Weidmann (2011) model both relocation and escalation diffusion through a joint count statistic. However, they use gridded units for their analysis, and therefore rely on aggregation to areal units. Additionally, although Loeffler and Flaxman (2017) present a strong analysis of diffusion of crime over continuous space, they do not differentiate between these two kinds of diffusion presented by Schutte and Weidmann (2011) or other characteristics of events that are critical to understanding conflict. Therefore we are interested in how the log-Gaussian Cox Process (LGCP) model framework may be parameterized to model diffusion of conflict data, with various characteristics of events that are crucial to understanding the diffusion of conflict events.

\subsection{Grid Models}

We continue our review of related work by studying the use of grid models to motivate why continuous space models are necessary and feasible to the study of conflict events. In past research, grid squares were often used as the unit of analysis because it allows researchers to specify the scale of the analysis based either on a theoretical framework of expected conflict distributions or on available sub-national data. Gridded squares have been increasingly used in empirical conflict studies due, in part, to the fact that aggregate-level administrative or other types of areal units, 
such as counties/regions or Census tracts, can mask conflict dynamics (Fjelde and Hultman, 2014; Dittrich Hallberg, 2012; Wood and Sullivan, 2015). Furthermore, the use of sub-state conflict event data offers the opportunity to explore the local distribution of violence over time.

For example, the PRIOGRID data set is an aggregate format of global-scale disaggregate (geo-coded point level) armed conflict events (Tollefsen et al., 2016). PRIOGRID counts of conflict events are the sums of point level conflict events that happened within a given spatial grid cell. This data set has contributed to the emergence of many new studies (Von Uexkull et al., 2016; Theisen et al., 2012; O'Loughlin et al., 2012; Hendrix and Salehyan, 2012). Nonetheless, when using gridded data, the Modifiable Areal Unit Problem (MAUP) represents a significant challenge where the level of aggregation can influence the results of the statistical analysis. The MAUP can significantly weaken and bias a statistical result when smaller areal units are aggregated to form larger areal units. Under this scenario, rezoning or moving boundaries of an area can have significant effects on the results (Wong, 2009).

There is little to no agreement across studies in terms of existing empirical findings using grid cells as the units of analysis, nor can this modeling strategy be used to capture the complex spatial dynamics and diffusion of conflict processes because it is limited by its aggregated nature to a certain resolution of analysis and accuracy. Therefore, we turn our attention to continuous space models where we do not rely on aggregation to areal units, such as grid cells.

\subsection{Continuous Space Models}

There are few empirical works in conflict studies that analyze conflict events over continuous space. Zammit-Mangion et al. (2012) provides one of the first attempts to model conflict, instead of crime or disease diffusion, over continuous space. However, in their model they do not specifically discuss the parameterization of diffusion, although they suggest it is possible to model diffusion using their model. Instead, they measure volatility and heterogeneous growth and give explicit parameterizations for each. They use a continuous space and discrete time version of the log-Gaussian Cox Process (LGCP) model, as they recognize that conflict data is reported on the day level, instead of at the exact time. They use the Stochastic Integro-Differential Equation (SIDE) approach to estimate the effects of time. The main focus of their study is on prediction, and they did not focus on the interpretations of the parameters.

There is some existing work on measuring diffusion of other types of events in continuous space, where the authors use the Hawkes process to model crime for example (Loeffler and Flaxman, 2017). However, in Loeffler and Flaxman (2017), they study gun crimes, which is a classical example of a point process in which the event happens in an exact instance in time. As stated above, in conflict data the event happens over the span of a day, or sometimes several days or weeks. Therefore, in 
our approach, we also analyze the difference in diffusion dynamics between events that last longer than 1 day as compared to events that only last 1 day.

We characterize conflict diffusion mechanisms in continuous space across South Sudan for the five types of conflict events discussed above, based on the duration of conflict events and military power of actors involved in conflicts. Thus far, researchers studying diffusion processes of conflict and some other social phenomena, such as crime, often use areal unit models, where events and other kinds of data are aggregated to usually administrative areas or grid cells by either the data provider or the researcher. Therefore, even when point or event level data is available, these processes are not frequently studied in continuous space. Though there have been some studies of conflict events in continuous space (Zammit-Mangion et al., 2012), none has specifically parameterized diffusion in conflict event data in continuous space. Therefore, we tackle the challenge of modeling spatial dependence of conflict events in continuous space.

\section{Data}

Event or point level data is characterized by an exact location, or coordinates, affiliated with the event and the events sometimes also have date/time affiliations. We refer to these events as points as these will be the "points" in our "point process" statistical methodology and they occur at exact points in space. Using data from the Armed Conflict Location and Event Data (ACLED) (Raleigh et al., 2010), this study examines conflict processes of five event types, including three types of battles (in which armed forces are often involved with different outcomes of territorial integrity), riots/protests (public demonstration and violent protest), and violence against civilians (physical harm to civilians, which can also be committed by rioters and/or to protesters) in South Sudan from 2011 to 2018. ACLED collects this data through news from international, regional, and local media reports as well as NGO accounts (ACLED, 2019).

Our full dataset consists of 4654 conflict events in South Sudan from July 14, 2011 through December 10, 2018. We see in Fig. 1 that there are many more conflict events that occur after 2014. Figure 1 also illustrates the event type over time, where most of these events are battles where there is no change in territory and violence against civilians. The counts of each of these event types for the full time period are presented in the legend of Fig. 1.

In Fig. 2a, we see the spatial distribution of these 4654 events over the country of South Sudan, where most of the events occur in the middle of the country. Each event has an affiliated transparency, so where there are darker colors in Fig. 2a and b, that means there are more events occurring at that location.

In the ACLED dataset, each day of an event is given a separate row in the dataset. If we count each of the days of a given conflict as separate events, then we have a total of 4654 conflict events as stated earlier. However, if we treat events as the same if they share the same location and description and occur within similar time periods, 


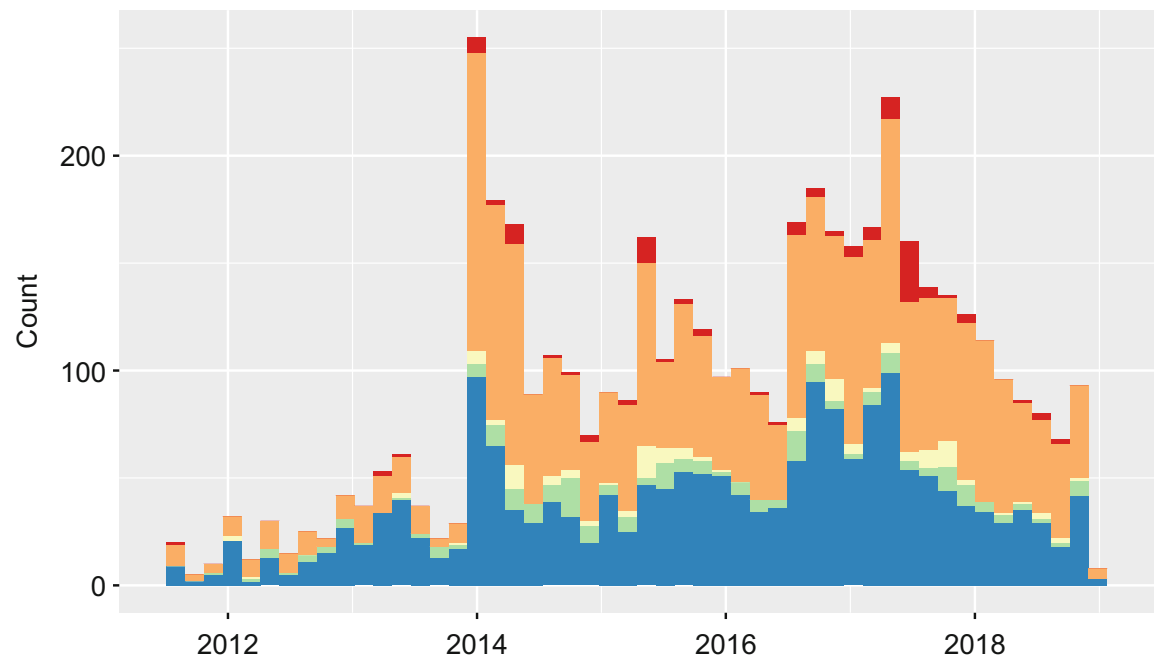

Event Type (Total Count, 2011-2018)

Battle-Government regains territory (126)

Battle-Non-state actor overtakes territory (137)

Battle-No change of territory $(2,290)$

Riots/Protests (249)

Violence against civilians $(1,852)$

Fig. 1 Frequency of conflict events in South Sudan over time, by conflict event type. The histogram bars are stacked on top of each other by event type

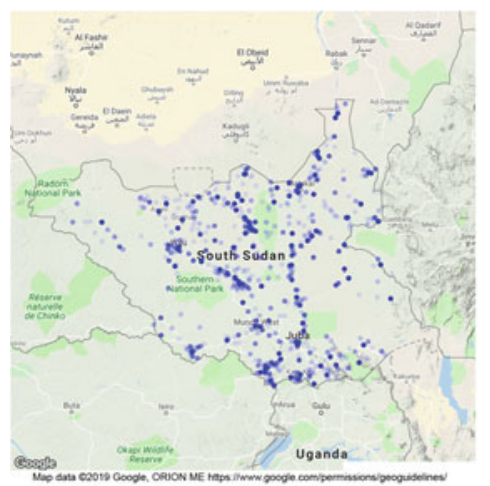

(a)

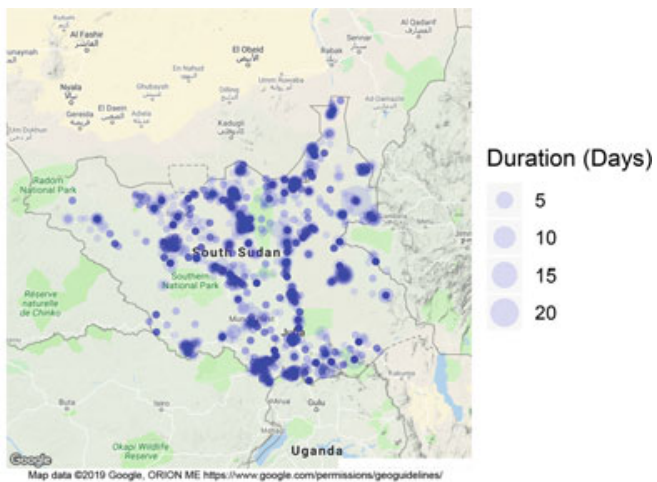

(b)

Fig. 2 Spatial distribution of conflict events over South Sudan. In Fig. 2a, all points, which represent conflict events, are equal size and the darker the point, the more events have occurred at that location. In Fig. 2b, the size of the point represents the duration of the event, and the shading again represents more events at that location. (a) Location only. (b) With duration

and then assign the duration as the number of days that the event persists, then we only have 4160 unique events in our dataset. When duration is defined in this way, the duration of an event ranges from 1 day to 24 days. Most events last 1 day with 
Table 1 We show common actors by frequency of conflict event involvement in South Sudan. We include the actor type that we assigned to each of these actors, in order to provide examples of actors in each category

\begin{tabular}{l|r|l}
\hline Actor name & Frequency & Actor type assigned \\
\hline Military Forces of South Sudan (2011-) & 2401 & State \\
\hline Civilians (South Sudan) & 1719 & Civilian \\
\hline Sudanese Peoples Liberation Army/movement-in opposition & 1536 & Non-state \\
\hline Unidentified Armed Group (South Sudan) & 989 & Non-state \\
\hline Police Forces of South Sudan (2011-) & 190 & State \\
\hline Protesters (South Sudan) & 177 & Civilian \\
\hline Murle Ethnic Militia (South Sudan) & 161 & Non-state \\
\hline Unidentified Communal Militia (South Sudan) & 156 & Non-state \\
\hline Mutiny of Military Forces of South Sudan (2011-) & 144 & State \\
\hline Rioters (South Sudan) & 99 & Civilian \\
\hline
\end{tabular}

the mean duration being 1.11 days. In Fig. 2b, we see the duration of these events illustrated by the size of the point. Figure $2 \mathrm{~b}$ gives a sense of the spatial distribution of the events that last longer than 1 day. For the purposes of our analysis, due to the fact that the vast majority (93\%) of conflict events only last 1 day and we would like to treat these events to be independent, we take the first day of the given conflict event and assign each event their duration as a covariate. Therefore, our final dataset for modeling purposes consists of 4160 unique events all of which are assigned their duration as a characteristic of the event, which we will analyze in detail later.

Through the ACLED dataset, we have two actors that are included with most events. There are no events that are missing the first actor and there are only 192 out of the 4160 events in our final dataset that are missing a second actor, i.e., where there was only one actor involved. These are exclusively events where there are only rioters or protesters involved in the event. There may be events with more than two actors involved, but ACLED only provides the first two actors involved. From these 4160 events, we have 285 unique actors in our dataset. We see some of the most commonly occurring actors below in Table 1, along with their assigned actor type.

As mentioned in Sect. 1, it is important to consider the difference in diffusion mechanisms between events that include state and non-state actors as well as civilians/rioters/protesters. To address this difference, we re-code and divide all of the ACLED actors into state actors, non-state actors, and civilians/rioters/protesters. We abbreviate the last category as simply "civilians" going forward. State actors include governments, governmental agencies (e.g., security department or police department), and the military force formed by a government or ruling party. Non-state actors contain a wide range of rebel groups, including identity militias and political opposition organizations. The last group includes civilians, rioters, protesters, and prisoners. The main difference between non-state actors and this category of civilians/rioters/protesters/prisoners is that non-state actors are organized actors with military power and the civilian groups have no military power. 
Table 2 We show the counts of conflict events by the actor dyads that were involved for South Sudan from 2011 to 2018. We also provide an example of an actor dyad for each dyad type. For the case of only civilian involvement, these events often did not include a second actor

\begin{tabular}{l|l|l}
\hline Actors involved & Frequency of event & Example \\
\hline State and non-state & 1556 & Yau Yau Rebels, Government of South Sudan \\
\hline Non-state and civilian & 1161 & Dinka Ethnic Militia, civilians \\
\hline State and civilian & 579 & Military Forces of South Sudan, civilians \\
\hline Only non-state & 415 & Kuei Ethnic Militia, Rup Dinka Ethnic Militia \\
\hline Only state & 230 & $\begin{array}{l}\text { Military Forces of South Sudan, Police Forces of } \\
\text { South Sudan }\end{array}$ \\
\hline Only civilian & 219 & Protesters (one actor) \\
\hline
\end{tabular}

After this categorization, we then assess the difference in diffusion of conflict events that include different combinations of these kinds of actors, including only state actors, only non-state actors, or only civilians as well as the dyads of these actor types. In Table 2, we show the number of conflict events that fall under each actor dyad. We see that most events involve either non-state and state actors or nonstate actors and civilians. We note that at times, as mentioned earlier, there is only one actor involved, which would necessitate this being categorized as only state, non-state, or civilians. However, this only occurs in $4.6 \%$ of the events. An example for each kind of event is included below in Table 2.

\section{Analysis}

\subsection{Test for Complete Spatial Randomness}

To begin our analysis, first we test for complete spatial randomness, or if our point process of conflict events follows a homogeneous Poisson Process. We perform this test because if the data follows complete spatial randomness, then there is no need for more complicated statistical testing. We define a homogeneous Poisson Process as follows:

Definition 4.1 Homogeneous Poisson Process: Let $N(A)$ denote the number of events in a region $A$, and $|A|$ the area of this region, then the data/events $X_{1}, X_{2}, \ldots, X_{N(A)}$ follow a homogeneous Poisson Process if the following conditions are fulfilled:

1. For some $\lambda>0$ and any finite region $A, N(A) \sim \operatorname{Poisson}(\lambda|A|)$

2. Given $N(A)=n$, the $n$ events form an independently and identically distributed (iid) sample from the uniform distribution on $A$.

3. For any two disjoint regions $A$ and $B$, the random variables $N(A)$ and $N(B)$ are independent. 
If our data follows a homogeneous Poisson Process, then it also follows complete spatial randomness (CSR). The parameter $\lambda$ is the rate or the intensity of the point process. Before we proceed to build more complicated models, we will test the null hypothesis of complete spatial randomness. We diagnose CSR through Monte Carlo sampling and empirical cumulative distribution functions (ECDF's), which we define below.

Definition 4.2 Empirical cumulative distribution function (ECDF): If $X_{1}, \ldots, X_{n}$ are iid with $\operatorname{CDF} F$, then the ECDF is $\hat{F}(x)=\frac{\sum_{i=1}^{n} \mathbb{1}\left\{X_{i} \leq x\right\}}{n}=\frac{\#\left\{X_{i} \leq x\right\}}{n}$. This is an unbiased estimator of $F(X)=P\left(X_{i} \leq x\right)$.

For our use of the ECDF, in this approach, we use Monte Carlo sampling to construct simulation envelopes under CSR. We sample $x_{1}, x_{2}, \ldots x_{n}$ locations uniformly on A and construct $\hat{F}$ as defined above. We use empty space distances where $d(u)=\min _{i}\left\|u-x_{i}\right\|, i \neq j$. We then construct our estimate $\hat{F}_{u}(r)$ of $F_{u}(r)=P(d(u) \leq r)=P($ at least one point within radius $r$ of $u)$. We then compare $\hat{F}_{u}(r)$ to $F_{u}(r)$ under CSR.

We conduct this test for CSR and plot the estimates for the F function, the simulation estimate, and the observed $\mathrm{F}$ function below in Fig. 3. In these tests, if we see that the observed line is very close to the mean simulated line, then we have evidence for CSR. If the observed line lies outside of the simulation envelope, or the dark shaded area, then we have evidence against CSR. For the pointwise case, the simulation envelopes, or the shaded areas, are constructed by sorting the simulated values and taking the $m$ th lowest and $m$ th highest values (Baddeley et al., 2015). For the simultaneous case, the simulation envelope is slightly more complicated. For each simulation, we calculate the theoretical mean value of $F$ under CSR and we calculate the maximum absolute difference between the theoretical curve and the simulated curve. After the simulations, we take the $m$ th largest absolute deviation from all the simulations, $d e v_{m}$, and this forms our simulation envelope through $l o=F_{\text {theo }}-d e v_{m}$ and $h i=F_{\text {theo }}+d e v_{m}$ where $F_{\text {theo }}$ is the theoretical mean value (Baddeley et al., 2015). We use two different techniques to estimate the F function: simultaneous and pointwise. We use both the simultaneous and pointwise method because through the use of the simultaneous envelopes, we get a smoother function but a much more conservative estimate of CSR as compared to the pointwise envelopes. In both tests, we have 800 simulations and choose $m$ to be 20 .

In Fig. 3, we see the estimates of the F function for both the pointwise and simultaneous methods. We see that even for the more conservative method, the simultaneous envelope, we see strong evidence against complete spatial randomness (CSR), as the observed curve is not close to the mean simulated curve under CSR. Therefore, we conclude that spatial modeling is appropriate for our dataset. 

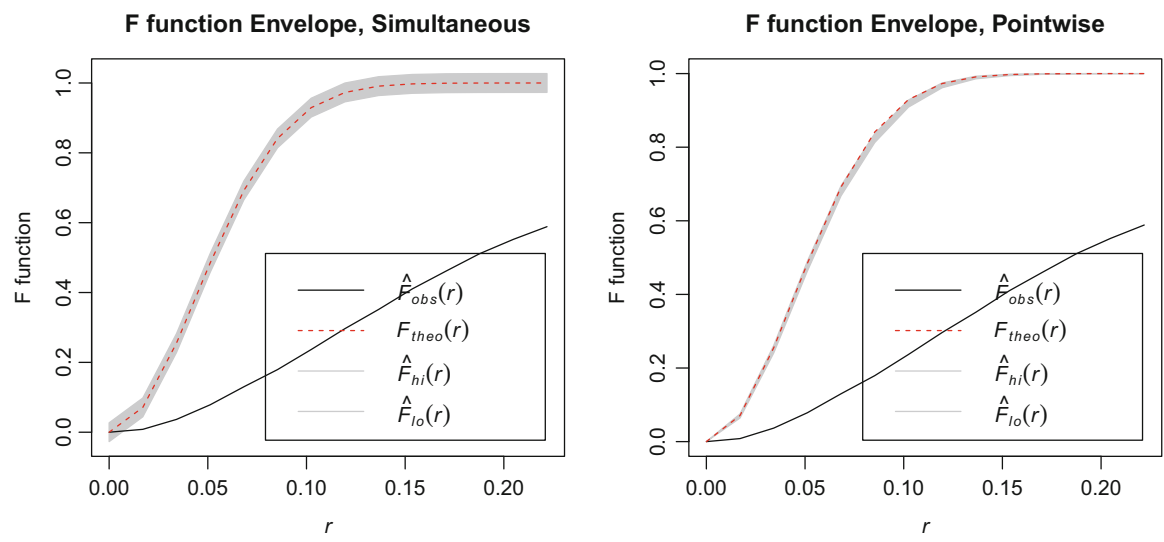

Fig. 3 We diagnose the absence of complete spatial randomness (CSR) through the simultaneous and pointwise simulation envelopes, as the observed curve lies outside of the envelope of the simulated curve

\subsection{Continuous Space Model}

Now that we have established that spatial modeling is appropriate, as the data does not follow complete spatial randomness, we start with a simple continuous space model. First, we treat our dataset as an inhomogeneous Poisson Process with an intensity $\lambda(x)$. We create a kernel density estimate for the intensity $\lambda(x)$ over space and time (Rowlingson and Diggle, 1993). First, we plot the estimate of the spatial intensity over the complete time window as well as the estimate for the temporal trend over the full area through a quartic kernel. In Fig. 4a, we see a high estimate for the kernel density estimate in the southern part of the country, as well as a couple of other peaks. In regards to the time dimension in Fig. 4b, the low count of conflict events at the end of the time window is due to lack of data in the last month of the dataset, as the dataset only covers through mid-December. We also see a low count for the number of events at the beginning of the time window, due to the actual low frequency of events from 2011-2014, as described earlier. The difference between these two low counts at the beginning compared to the end is due to observed low frequencies vs lack of data availability in the time window, respectively. We see a decrease and then increase in conflict in the middle of the dataset.

Next, we plot the estimate of the intensity function over 12 month intervals for our 8 years of data. We note that although time is continuous, we discretize time into years in order to visualize the change in the kernel density estimate over time. In Fig. 5, we see that our spatial kernel density estimate changes over the year-long periods. In Fig. 5a, all of the kernel density estimates use the same scale so we are able to see that there is really only one time period, in 2016, where the conflict events are quite concentrated in space at high levels in that concentration. Otherwise, they are pretty evenly distributed throughout the country at lower levels than 2016. In 


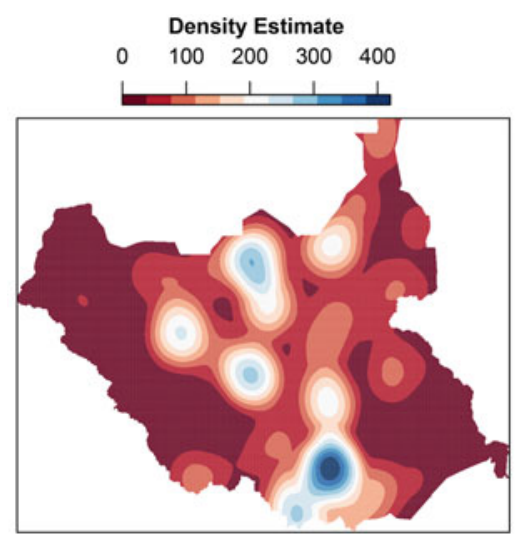

(a)

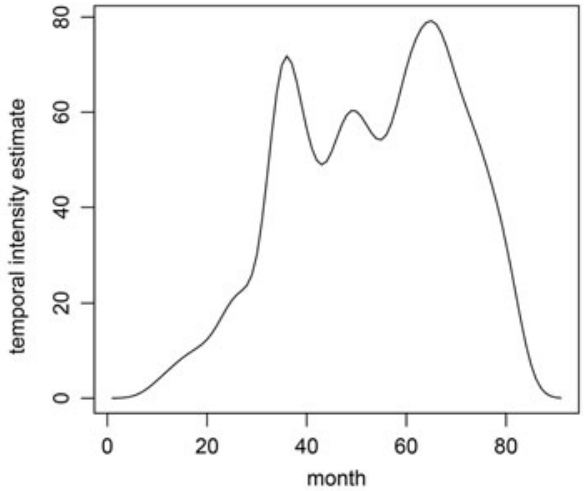

(b)

Fig. 4 Through a kernel density estimate for the spatial intensity function, $\lambda(x)$, and the temporal intensity function, we see an estimate of the spatial distribution and temporal trend of conflict events from 2011-2018. (a) Spatial intensity function estimate. (b) Temporal intensity function estimate

2011

(a)

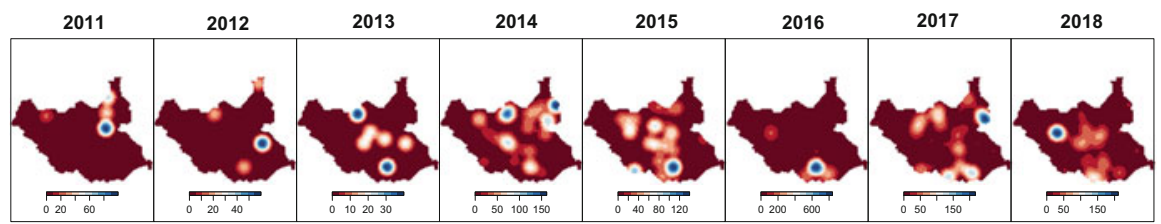

(b)

Fig. 5 Through the kernel density estimate of the intensity function $\lambda(x)$ over space and time, we see how the spatial distribution of conflict events changes over each year in our dataset. Through the common scale, we see there is only 1 year, 2016, with a strong spatial peak relative to the other time intervals. However, if we use a scale determined by each year, we can see where the conflict events are spatially concentrated by year. (a) Common scale. (b) Scale by year

Fig. 5b, we have a different color scale for each year of the data, so you can see the relative peaks within the given year of data and where the spatial peaks are shifting. Specifically, we see that there is a region in the southern part of South Sudan that often has fairly high conflict rates over the full 8 year conflict window. 
However, there are other areas in the country, such as in the north east, which have high conflict events for some periods but not others.

\subsection{Gaussian Process}

Now that we have seen a preliminary estimate of the intensity function, we will model our continuous space data as a Gaussian Process in order to study diffusion mechanisms in the conflict process. We do this through fitting a log-Gaussian Cox Process (LGCP) through the spatstat package in R (Baddeley et al., 2015). In an LGCP model, the intensity function is defined as $\lambda(x)=\exp (Z(x))$ at location $x$ where $Z(x)$ is a Gaussian random field in the two-dimensional plane (Moller and Waagepetersen, 2003). The intensity of the LGCP is then governed by the Gaussian Process, $Z(x)$, which has covariance function $C(r)$ where $r$ is the distance between two points. We use an exponential covariance function so that the covariance function takes the following form:

$$
C(r)=\sigma^{2} e^{-r / \alpha}
$$

Through the spatstat package in $\mathrm{R}$, we estimate $\sigma^{2}$ and $\alpha$ for our data. We plot the estimate of the covariance function to illustrate the spatial range of our data. Once the covariance function falls below a certain point, which we will specify as 0.1 , we call this distance the effective range of our events. The reason it is called range is because this is the distance at which events still impact each other with nonzero covariance. However, we will never actually observe a covariance of zero, so the reason this is called the effective range is due to the fact that the range is small enough, it is effectively 0 . A larger effective range suggests that events have a stronger influence or dependence on events for a larger radius around those given events. There is no inherent rule with the choice of the cutoff, as this is a heuristic process. However, the covariance function is most accurate when the radius is small. Therefore, when we specify a smaller cutoff, we are effectively choosing a larger radius where the covariance function is not as accurate. In the figures in this section, the dashed line represents the cutoff point of 0.1 .

We note that the number of events in each category does not necessarily affect the value we find for the effective range. In other words, a smaller number of events in one category does not necessarily lead to a higher or lower estimate of our effective range. However, a smaller number of events in the category could affect the precision of our estimate, which we will address in Sect. 5.

First, we compare the effective spatial range for each year of the conflict data in order to make conclusions about the change in diffusion mechanisms over time. In Fig. 6, we plot the effective range through the covariance function estimate for each year. We see that the estimate of the covariance function does change over time in our dataset, but not drastically. We include the effective range over time in Table 3. We see that the effective range decreases from 2011 to 2014 and then increases from 


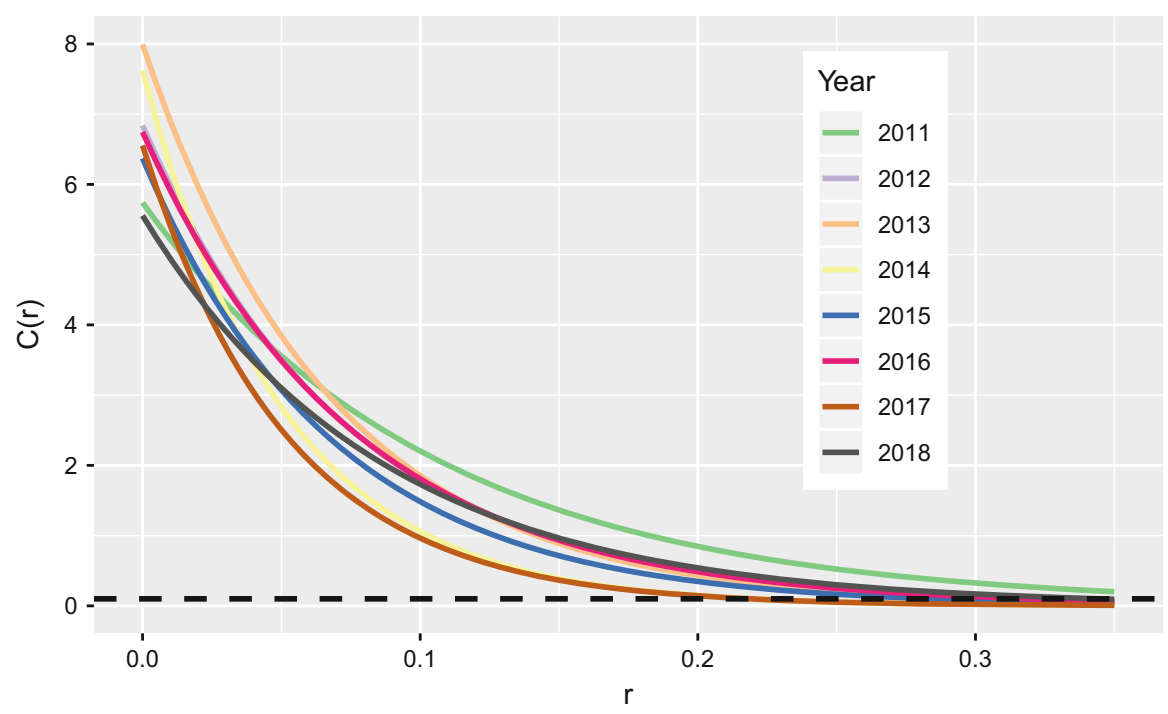

Fig. 6 When we plot the estimate of the covariance function by year, we see lower estimates of the effective range for 2014, 2015, and 2017

Table 3 Effective range

estimates by year

\begin{tabular}{l|l}
\hline Year & Effective range \\
\hline 2011 & 0.42 \\
\hline 2012 & 0.32 \\
\hline 2013 & 0.30 \\
\hline 2014 & 0.22 \\
\hline 2015 & 0.28 \\
\hline 2016 & 0.32 \\
\hline 2017 & 0.22 \\
\hline 2018 & 0.34 \\
\hline
\end{tabular}

2014-2016 but this increasing trend does not hold through 2018. This implies that between 2011 and 2014, events started to exhibit a weaker dependence structure, where events had a smaller effect on surrounding events than previously. This makes intuitive sense as conflict events were relatively infrequent in the region throughout this time period. Between 2014-2015, the effective range increased, meaning that each event had a stronger effect for more distance, or a larger radius, $r$, around that given event. This also makes intuitive sense as there were large increases in conflict events during this time period. As stated earlier, there is not necessarily a relationship between more events in a given conflict event group and a higher or lower effective range. However, more events occurring in a certain period of time may suggest that these events would spur more events following them and have a stronger dependence mechanism, and would therefore have a larger effective range. The smaller effective range may also be due to the fact that there are multiple 


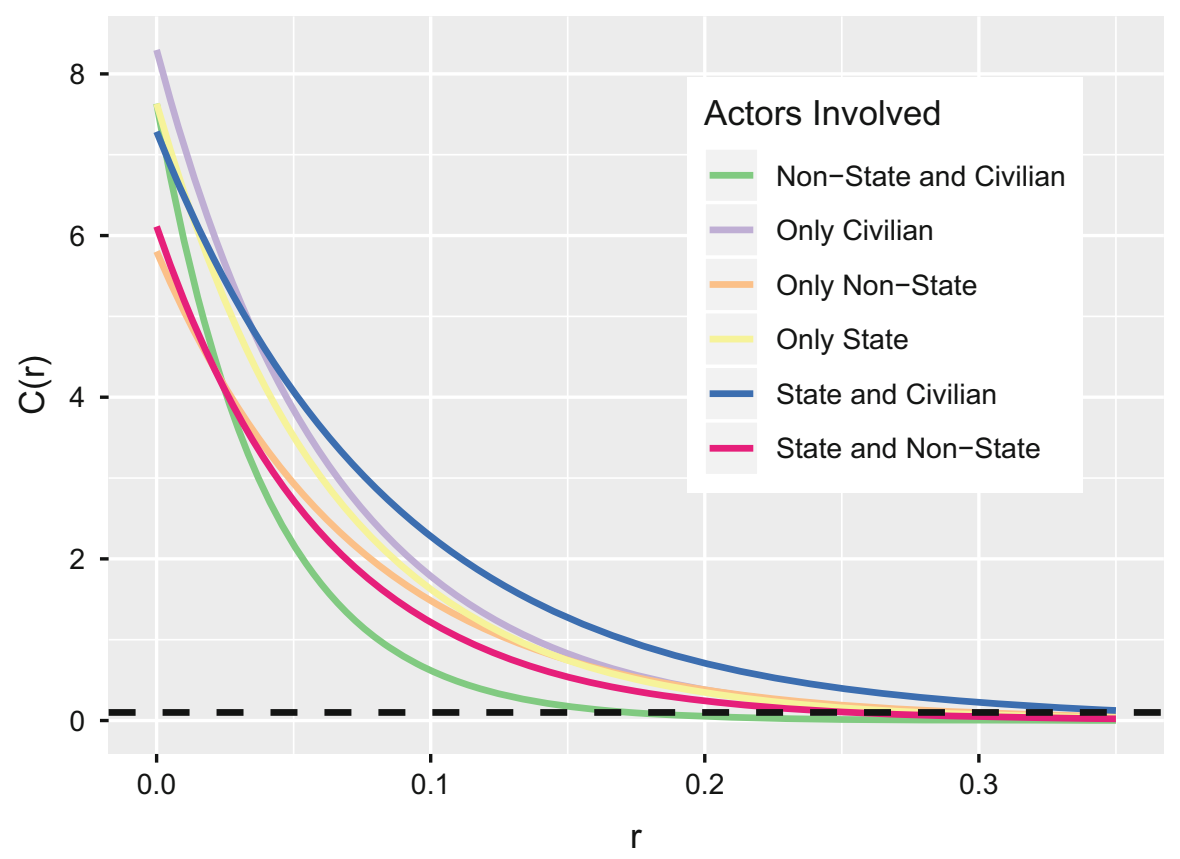

Fig. 7 When we plot the estimate of the covariance function by actor type, we see higher estimates for the effective range for the state actor and civilian dyad and a small effective range when there are non-state actors and civilians involved

Table 4 Effective range estimates by actors involved

\begin{tabular}{l|l}
\hline Actors involved & Effective range \\
\hline Only state & 0.28 \\
\hline Only non-state & 0.30 \\
\hline Only civilian & 0.29 \\
\hline State and non-state & 0.25 \\
\hline State and civilian & 0.37 \\
\hline Non-state and civilian & 0.17
\end{tabular}

clusters of conflict events during these years (2013, 2014, 2015, and 2017), as seen in Fig. 5b.

Next, we compare the effective range of conflict events based on the types of actors that are involved. We see in Fig. 7 and Table 4 that the effective range is highest for events where state actors and civilians are involved. The effective range is quite similar for other conflict events, especially as the radius gets higher. This suggests that the diffusion mechanism might be strongest when there are state actors and civilians involved in the conflict but other actors do not have a large effect on diffusion. This would illustrate that when state actors and civilians are involved, this may spur additional events around it more-so than other conflict events, perhaps due to the contentious nature of these kinds of events. 


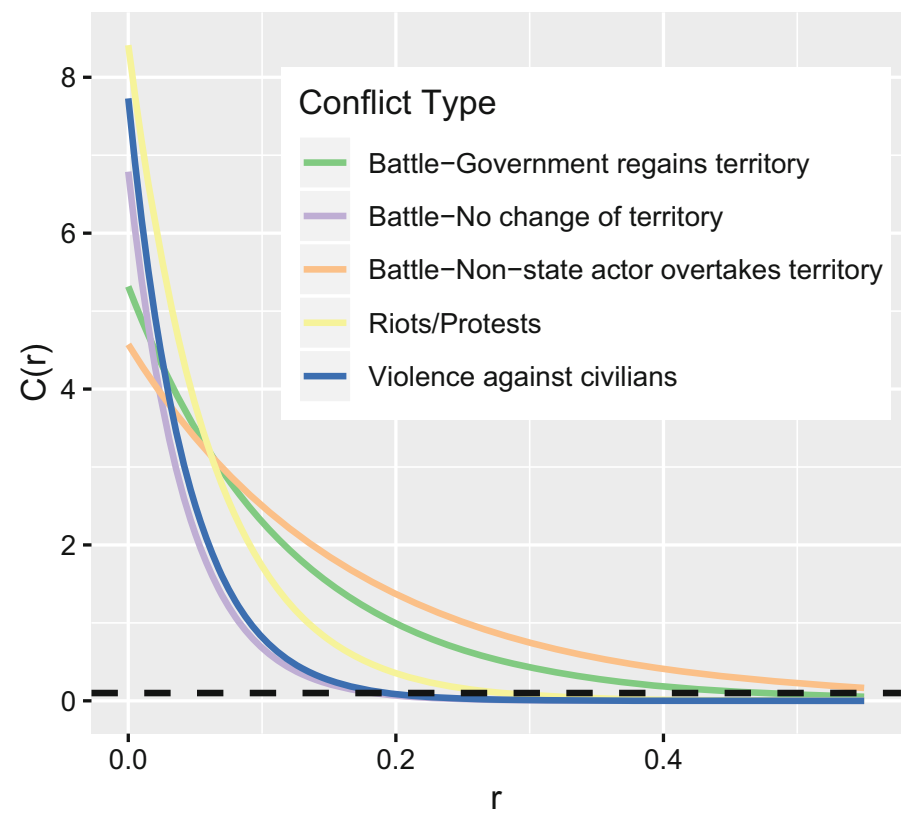

Fig. 8 When we plot the estimate of the covariance function by conflict type, we see a larger effective range for battles where non-state actors overtake territory or when government regains territory

Table 5 Effective range estimates by conflict type

\begin{tabular}{l|l}
\hline Conflict type-specific & Effective range \\
\hline Violence against civilians & 0.195 \\
\hline Riots/protests & 0.28 \\
\hline Battle-government regains territory & 0.475 \\
\hline Battle-no change of territory & 0.185 \\
\hline Battle-non-state actor overtakes territory & 0.635 \\
\hline
\end{tabular}

We also conduct this analysis based on conflict type. We see in Fig. 8 and Table 5 that the event types with the strongest diffusion mechanisms are battles where the government regains territory and battles where non-state actors overtake the territory. It is notable that battles with no change in territory have a relatively weak diffusion mechanism when compared to these other battle types, suggesting that it is necessary to differentiate between these kinds of conflict events. We thus see that these two types of battles, where there is change in territory, may impact the surrounding more than battles where there is no change in territory or violence against civilians. Riots and protests lie in the middle of these conflict event types.

Lastly, we also analyze these events by duration length. We show the difference in the diffusion mechanism for events that last only 1 day compared to events that last longer than 1 day. In Fig. 9 and Table 6, we see that events that last more than 1 day exhibit a much stronger diffusion mechanism through the larger effective range. 


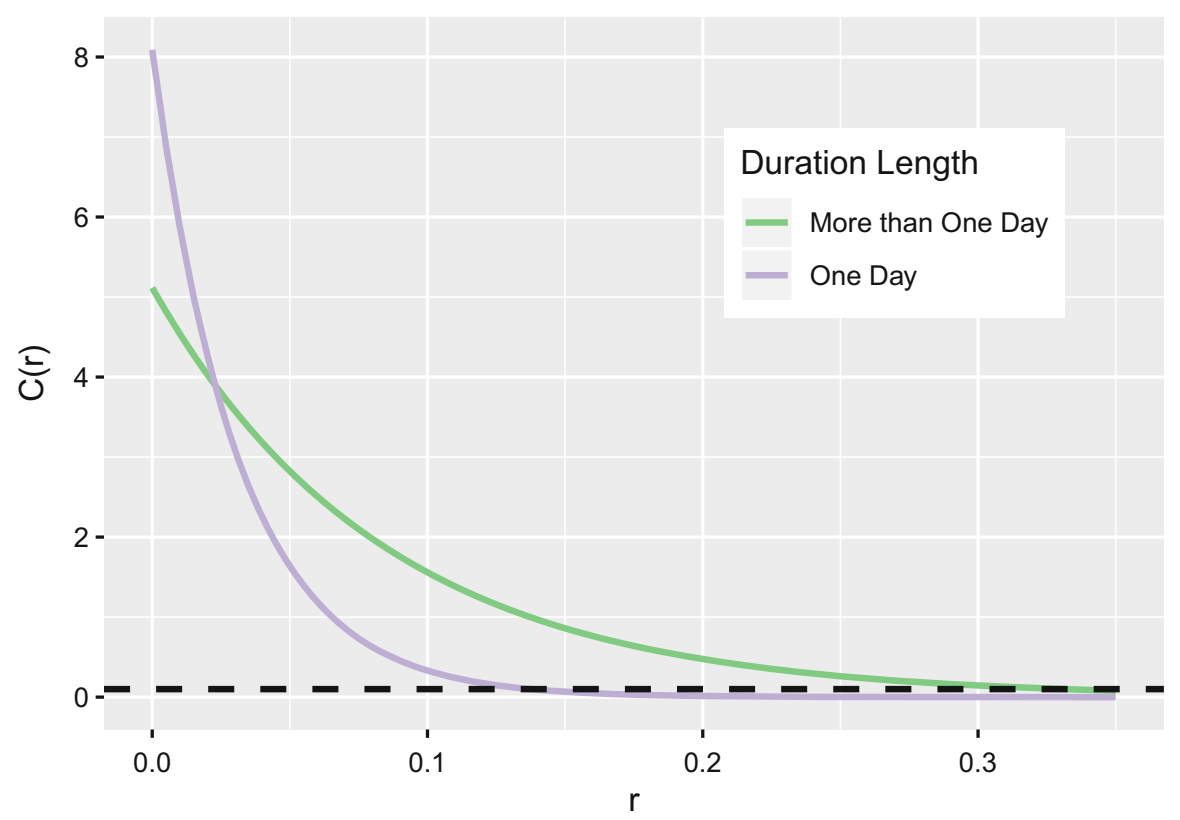

Fig. 9 When we plot the estimate of the covariance function by duration length, we see a larger effective range when the event lasts for longer than 1 day

Table 6 Effective range

estimates by duration length

\begin{tabular}{l|l}
\hline Duration length & Effective range \\
\hline One day & 0.14 \\
\hline More than 1 day & 0.335 \\
\hline
\end{tabular}

This makes intuitive sense as these longer events are likely to be larger-scale events in severity, and therefore might spur other events.

Through this analysis, we show that it is important to analyze diffusion mechanisms, such as the effective range of any given event, over time. We have shown that for our dataset, the dependence between events changes over time, and the effective range for conflicts in South Sudan decreased from 2011-2014, suggesting a weaker diffusion or dependence mechanism, but then increased from 2014-2016, as conflict escalated in the region. We have also shown that it is important to take into consideration event types, actor types, and duration of the event when considering diffusion mechanisms between events.

\section{Discussion and Future Work}

Our results illustrate that it is valuable to characterize the dependence between events when we have rich data in continuous space. We began by characterizing 
the dependence over time and space through density estimates and formally estimated this dependence through a log-Gaussian Cox Process (LGCP) model and the covariance function. We found that it is important to estimate changes in the diffusion mechanism over time and across actors and conflict types to detect differences in the diffusion process.

The theoretical implication of our empirical analysis is two-fold. First, battles with territorial gains for one side tend to diffuse over larger distances than battles with no territorial change. This implies that the location of an individual conflict event and the clustering of multiple events or locations of events could have significant effects on the subsequent onset or termination of other conflict events. Based on our analysis, the southern part of South Sudan, which is the area around the capital, Juba, has a higher estimated intensity than other surrounding regions. A higher estimated conflict intensity around the southern part of South Sudan is evidence of the spatial interdependence among conflict events. This result reflects a historical conflict diffusion process of mass killings of the Nuer people by Dinka paramilitary groups in the capital, Juba, which were the pretext to the origin of the outbreak of civil war (Sawe, 2017). In the future, we would also like to control for certain demographic and socioeconomic characteristics over space and time, including population, housing, and transportation infrastructure.

Second, modeling spatio-temporal distribution of conflict events will contribute to defining the conjuncture of initial conditions of locations where conflict took place and capturing plausible mechanisms underlying changes on the spatial distribution or pattern of conflict over times. Our analysis shows that conflicts with longer duration exhibit stronger spatial dependence. Throughout the process of being an independent country, conflict events in South Sudan are most spatially concentrated in 2016, when compared to the other years in our dataset. This finding reveals that the long-term unsolved ethnic tension between the government and rebel groups led to the outbreak of large-scale violence against civilians in 2016. Both the government and the rebel groups committed abuses against civilians in and around Juba and other areas. According to the Global Conflict Tracker and Human Rights Watch, the government and the rebel group targeted civilians along ethnic lines, and millions of people were displaced or forced to flee their homes. Military groups committed rape and sexual violence, destroyed property and looted villages, and recruited children into their ranks. The UN even warned that this ongoing ethnic war was likely to transform into genocide (GlobalConflictTracker, 2019; HumanRightsWatch, 2017). Therefore, in future research, it is crucial to investigate how ethnic identity might service as an underlying mechanism reshaping the spatial patterns of conflict.

Third, we have shown that it is important to consider several key characteristics of conflict events when analyzing the dependence structure of these events. For example, we show that temporal analysis, rather than simply a cross-section, may be important in these analyses, which agrees with existing literature that also studies temporal dynamics (Read and Mac Ginty, 2017; Silwal, 2013). This temporal analysis should be expanded in future work. We also illustrate that the types of actors that are involved with conflicts can play a crucial role in determining if that 
event is likely to influence other events. Specifically, we find that when state actors and civilians/rioters/protesters are involved, this is likely to have a stronger effect on surrounding events, which bridges a gap in the literature (Cunningham et al., 2013). We also find that it is important to consider event types, as battles where there are changes in territories have stronger diffusion mechanisms than other types of conflict events. This adds to the literature, as most studies do not investigate diffusion mechanisms between the types (Wood, 2010; Azam and Hoeffler, 2002; Balcells, 2010). Lastly, we find that events that last only 1 day vs longer than 1 day also have quite different dependence structures, as events that last longer than 1 day have much stronger dependence on events surrounding them. To the best of our knowledge, this study is the first that empirically examines duration using the dayspan as the unit of analysis and carefully examines whether the events that persist only 1 day are important. Our results were gathered through the use of continuous space models and point level data, and therefore represent an advancement on most existing methods.

There are some caveats that are necessary in this analysis. Most importantly, in the estimate of our covariance function, there are more points at a smaller radius around a point and less points once you get further away. Therefore, as we get to a larger radius, the covariance estimate is less reliable and we interpret the difference in the covariance function with some caution at larger distances. As mentioned earlier, if there are fewer events in a given category, the precision of the covariance function estimate, and therefore also the effective range estimate, may be affected. One way to assess the variability of these estimates is through a parametric bootstrap where we would simulate multiple realizations from a fitted model and assess the variability in the resulting estimates of the effective range. However, given the computationally intensive nature of these models, we interpret our results with caution, only draw conclusions where there are large differences in the effective range estimates, and suggest this for future work.

In the future, we could consider different formal models for diffusion mechanisms, through the epidemiology literature. However, the log-Gaussian Cox Process methodology provides an easily interpretable mechanism to analyze diffusion in the context of conflict event data. We would also like to incorporate stronger temporal measures of dependence into our analysis, such as through continuous time models, as well as covariate information. An analysis by conflict severity, based on the number of causalities in addition to our analysis of severity by duration of the conflict, could also be an additional important step in this analysis.

Acknowledgements The authors would like to acknowledge Dr. Murali Haran for his helpful feedback throughout the planning stages of this chapter. This work was supported by the National Science Foundation under IGERT award DGE-1144860, "Big Data Social Science." 


\section{References}

ACLED (2019). Armed conflict location and event data project (ACLED) codebook, 2019.

Anselin, L., \& Baltagi, B. (2001). A companion to theoretical econometrics. In Spatial econometrics (pp. 310-330). Oxford: Blackwell.

Anselin, L., \& O'Loughlin, J. (1990). Spatial econometric analysis of international conflict. In Dynamics and conflict in regional structural change (pp. 325-345). Berlin: Springer.

Azam, J.-P., \& Hoeffler, A. (2002). Violence against civilians in civil wars: Looting or terror? Journal of Peace Research, 39(4), 461-485.

Baddeley, A., Rubak, E., \& Turner, R. (2015). Spatial point patterns: Methodology and applications with $R$. London: Chapman and Hall/CRC Press.

Balcells, L. (2010). Rivalry and revenge: Violence against civilians in conventional civil wars. International Studies Quarterly, 54(2), 291-313.

Balcells, L. (2011). Continuation of politics by two means: Direct and indirect violence in civil war. Journal of Conflict Resolution, 55(3), 397-422.

Bera, A. K., Doğan, O., Taşpınar, S., \& Leiluo, Y. (2019). Robust LM tests for spatial dynamic panel data models. Regional Science and Urban Economics, 76, 47-66

Best, N. G., Ickstadt, K., \& Wolpert, R. L. (2000). Spatial poisson regression for health and exposure data measured at disparate resolutions. Journal of the American Statistical Association, 95(452), 1076-1088.

Braithwaite, A. (2010). Resisting infection: How state capacity conditions conflict contagion. Journal of Peace Research, 47(3), 311-319.

Buhaug, H., \& Gleditsch, K. S. (2008). Contagion or confusion? Why conflicts cluster in space. International Studies Quarterly, 52(2), 215-233.

Buhaug, H., Gleditsch, K. S., Holtermann, H., Østby, G., \& Tollefsen, A. F. (2011). It's the local economy, stupid! Geographic wealth dispersion and conflict outbreak location. Journal of Conflict Resolution, 55(5), 814-840.

Cederman, L.-E., \& Gleditsch, K. S. (2009). Introduction to special issue on "Disaggregating Civil war". The Journal of Conflict Resolution, 53(4), 487-495.

Collier, P., Hoeffler, A., \& Rohner, D. (2009). Beyond greed and grievance: Feasibility and civil war. Oxford Economic papers, 61(1), 1-27.

Crisis Group (2017). Instruments of pain (II): Conflict and famine in South Sudan. Brussels: International Crisis Group.

Cunningham, D. E., Gleditsch, K. S., \& Salehyan, I. (2013). Non-state actors in civil wars: A new dataset. Conflict Management and Peace Science, 30(5), 516-531.

De Vries, L., \& Schomerus, M. (2017). South Sudan's civil war will not end with a peace deal. Peace Review, 29(3), 333-340.

Dittrich Hallberg, J. (2012). PRIO conflict site 1989-2008: A geo-referenced dataset on armed conflict. Conflict Management and Peace Science, 29(2), 219-232.

Fearon, J. D., \& Laitin, D. D. (2003). Ethnicity, insurgency, and civil war. American Political Science Review, 97(1), 75-90.

Fjelde, H., \& Hultman, L. (2014). Weakening the enemy: A disaggregated study of violence against civilians in Africa. Journal of Conflict Resolution, 58(7), 1230-1257.

GlobalConflictTracker (2019). Civil war in South Sudan: Global conflict tracker.

Harbom, L., Melander, E., \& Wallensteen, P. (2008). Dyadic dimensions of armed conflict, 19462007. Journal of Peace Research, 45(5), 697-710.

Hegre, H. (2014). Democracy and armed conflict. Journal of Peace Research, 51(2), 159-172.

Hegre, H., \& Nygård, H. M. (2015). Governance and conflict relapse. Journal of Conflict Resolution, 59(6), 984-1016.

Hegre, H., \& Sambanis, N. (2006). Sensitivity analysis of empirical results on civil war onset. Journal of Conflict Resolution, 50(4), 508-535.

Hendrix, C. S., \& Salehyan, I. (2012). Climate change, rainfall, and social conflict in Africa. Journal of Peace Research, 49(1), 35-50. 
Hoeffler, A. (2012). On the causes of civil war. In Oxford Handbook of the Economics of Peace and Conflict (Vol. 9, pp. 179-204). New York, NY: Oxford University Press.

HumanRightsWatch (2017). World report 2017: Rights trends in South Sudan.

Koc-Menard, S. (2006). Switching from indiscriminate to selective violence: The case of the Peruvian military (1980-95). Civil Wars, 8(3-4), 332-354.

Liang, S., Carlin, B. P., \& Gelfand, A. E. (2008). Analysis of Minnesota colon and rectum cancer point patterns with spatial and nonspatial covariate information. The Annals of Applied Statistics, 3(3), 943.

Loeffler, C., \& Flaxman, S. (2017). Is gun violence contagious? A spatiotemporal test. Journal of Quantitative Criminology, 34(3/4), 1-19.

Maves, J., \& Braithwaite, A. (2013). Autocratic institutions and civil conflict contagion. The Journal of Politics, 75(2), 478-490.

Moller, J., \& Waagepetersen, R. P. (2003). Statistical inference and simulation for spatial point processes. Boca Raton, FL: Chapman and Hall/CRC.

OCHA (2018). Humanitarian reports.

O’Loughlin, J., Witmer, F. D., Linke, A. M., Laing, A., Gettelman, A., \& Dudhia, J. (2012). Climate variability and conflict risk in East Africa, 1990-2009. Proceedings of the National Academy of Sciences, 109(45), 18344-18349.

Penghui, G., Lihu, L., \& Zhengming, Q. (2015). Robust test for spatial error model: Considering changes of spatial layouts and distribution misspecification. Communications in StatisticsSimulation and Computation, 44(2), 402-416.

Raleigh, C., Linke, A., Hegre, H., \& Karlsen, J. (2010). Introducing ACLED: An armed conflict location and event dataset, special data feature. Journal of Peace Research, 47(5), 651-660.

Read, R., \& Mac Ginty, R. (2017). The temporal dimension in accounts of violent conflict: A case study from Darfur. Journal of Intervention and Statebuilding, 11(2), 147-165.

Rowlingson, B. S., \& Diggle, P. J. (1993). Splancs: Spatial point pattern analysis code in S-Plus. Computers and Geosciences, 19(5), 627-655.

Sawe, B. E. (2017). Ethnic groups of South Sudan.

Schutte, S., \& Weidmann, N. B. (2011). Diffusion patterns of violence in civil wars. Political Geography, 30(3), 143-152.

Schwartz, R. A., \& Straus, S. (2018). What drives violence against civilians in civil war? Evidence from Guatemala's conflict archives. Journal of Peace Research, 55(2), 222-235.

Silwal, S. (2013). A spatial-temporal analysis of civil war: The case of Nepal. The Economics of Peace and Security Journal, 8(2).

Sparks, C. S. (2011). Violent crime in San Antonio, Texas: An application of spatial epidemiological methods. Spatial and Spatio-temporal Epidemiology, 2(4), 301-309.

Starr, H. (2003). The power of place and the future of spatial analysis in the study of conflict. Conflict Management and Peace Science, 20(1), 1-20.

Theisen, O. M., Holtermann, H., \& Buhaug, H. (2012). Climate wars? Assessing the claim that drought breeds conflict. International Security, 36(3), 79-106.

Toft, M. D. (2005). The geography of ethnic violence: Identity, interests, and the indivisibility of territory. Princeton, NJ: Princeton University Press.

Tollefsen, A. F., Bahgat, K., Nordkvelle, J., \& Buhaug, H. (2016). PRIO-GRID v. 2.0 codebook. Journal of Peace Research, 49(2), 363-374.

Valentino, B. A. (2014). Why we kill: The political science of political violence against civilians. Annual Review of Political Science, 17, 89-103.

Von Uexkull, N., Croicu, M., Fjelde, H., \& Buhaug, H. (2016). Civil conflict sensitivity to growingseason drought. Proceedings of the National Academy of Sciences, 113(44), 12391-12396.

Wang, H., Kifer, D., Graif, C., \& Li, Z. (2016). Crime rate inference with big data. In Proceedings of the 22nd ACM SIGKDD International Conference on Knowledge Discovery and Data Mining (pp. 635-644). New York, NY: ACM.

Ward, M. D., \& Gleditsch, K. S. (2002). Location, location, location: An MCMC approach to modeling the spatial context of war and peace. Political Analysis, 10(3), 244-260. 
Wong, D. (2009). The modifiable areal unit problem (MAUP). The SAGE Handbook of Spatial Analysis, 105, 23.

Wood, R. M. (2010). Rebel capability and strategic violence against civilians. Journal of Peace Research, 47(5), 601-614.

Wood, R. M., \& Sullivan, C. (2015). Doing harm by doing good? The negative externalities of humanitarian aid provision during civil conflict. The Journal of Politics, 77(3), 736-748.

Zammit-Mangion, A., Dewar, M., Kadirkamanathan, V., \& Sanguinetti, G. (2012). Point process modelling of the Afghan War Diary. Proceedings of the National Academy of Sciences, $109(31), 12414-12419$.

Open Access This chapter is licensed under the terms of the Creative Commons Attribution 4.0 International License (http://creativecommons.org/licenses/by/4.0/), which permits use, sharing, adaptation, distribution and reproduction in any medium or format, as long as you give appropriate credit to the original author(s) and the source, provide a link to the Creative Commons licence and indicate if changes were made.

The images or other third party material in this chapter are included in the chapter's Creative Commons licence, unless indicated otherwise in a credit line to the material. If material is not included in the chapter's Creative Commons licence and your intended use is not permitted by statutory regulation or exceeds the permitted use, you will need to obtain permission directly from the copyright holder. 